\title{
Large scale 3-D phase-field simulation of coarsening in Ni-base superalloys
}

\author{
Mohan Kumar Rajendran ${ }^{\text {a }}$, Oleg Shchyglo, and Ingo Steinbach \\ Interdisciplinary Centre for Advanced Materials Simulation (ICAMS), Ruhr University Bochum, Germany
}

\begin{abstract}
In this study we present a large scale numerical simulation of $\gamma-\gamma^{\prime}$ microstructure evolution in Nibase superalloy using the multi-phase field method in three dimensions. We numerically simulated precipitation hardening heat treatment cycles. Large scale three dimensional simulations are necessary in order to get sufficient statistics for predicting the morphological evolution, average $\gamma^{\prime}$ precipitate size, precipitates size distribution over time and ripening exponent for a given temperature and composition. A detailed analysis of obtained result is presented emphasising the effect of elastic interaction on the coarsening kinetics in Ni-base superalloy. The study is performed using the phase-field modelling library "OpenPhase" which is based on a multi-phase field multi-component model.
\end{abstract}

\section{Introduction}

Microstructure and its mechanical properties at elevated temperatures are the major factors controlling the performance of Ni-base superalloys. The microstructure typically found in superalloys consist of ordered intermetallic precipitates of $\gamma^{\prime}$ phase (L12 type FCC crystal structure) coherently embedded in Ni rich $\gamma$ matrix phase (also FCC crystal structure). The formation of $\gamma-\gamma^{\prime}$ microstructure is typically obtained during solution heat treatment and precipitation hardening processing cycles. During the $\gamma-\gamma^{\prime}$ phase transformation, elastic stresses arise due to lattice misfit among the phases. This in turn has a strong influence on the microstructure and its evolution, due to which the particles grow in a cuboid shape. The elastic interaction is significantly long-range, which causes strong spatial correlations between the precipitates, which align them along their soft directions. On a macroscopic scale, the mechanical properties such as yield strength, fatigue and creep resistance mainly depend on the control over two-phase $\gamma-\gamma^{\prime}$ microstructure evolution and $\gamma^{\prime}$ phase volume fraction.

High technological importance of superalloys led to significant efforts towards understanding the mechanisms responsible for $\gamma-\gamma^{\prime}$ microstructure formation over the past half century [1] and is still active in recent years [2-5]. At present there is a significant amount of knowledge related to prediction and modelling of microstructure evolution and coarsening kinetics. Lifshitz and Slyozov [6], and later Wagner [7] developed an analytical model for prediction of particles coarsening kinetics (LSW theory). They have proposed that the cube of the average particle size evolve linearly in time, while the normalized particle

a Corresponding author: mohan.rajendran@icams.rhuruni-bochum.de size distribution (PSDs) is time invariant. Their analytical prediction is important not only for design of commercial superalloys but also for understanding the effect of high service temperatures where coarsening and partial dissolution of $\gamma^{\prime}$ phase occur leading to material properties degradation.

Numerous experimental and simulation results show that the growth law of average particle size during coarsening follows the power law given in Eq. (1) with the coarsening exponent $\mathrm{m}$ close to 3 (Ostwald ripening exponent) for superalloys [5-9] and 2 for polycrystalline grain growth $[10,11]$

$$
\overline{\mathrm{A}}^{\mathrm{m}}-\overline{\mathrm{A}}_{0}^{\mathrm{m}}=\mathrm{kt} .
$$

Where $A_{0}$ is average cube length at the beginning of the coarsening, $\mathrm{k}$ is the coarsening rate constant.

The aim of the present work is the numerical study of the coarsening kinetics of ordered $\gamma^{\prime}$ phase precipitates in Ni-Al binary alloy using phase-field method in three dimension. In this study the heat treatment conditions are numerically simulated using the open-source phasefield simulation library "OpenPhase" [12] which is based on a multi-phase field multi-component model $[13,14]$. An effect of elasticity on the coarsening kinetics is investigated in detail.

\section{Simulation setup}

Most of simulations related to the microstructure formation in superalloys are mainly performed in two dimensions $[4,5,15]$. While 2D simulations allow to reproduce cuboidal (rectangular in 2D) shape of the $\gamma^{\prime}$ particles which is the main topological feature of the $\gamma-\gamma^{\prime}$ microstructure, it is not possible to study the realistic coarsening kinetics due to the intrinsic

This is an Open Access article distributed under the terms of the Creative Commons Attribution License 4.0, which permits unrestricted use, distribution, and reproduction in any medium, provided the original work is properly cited. 


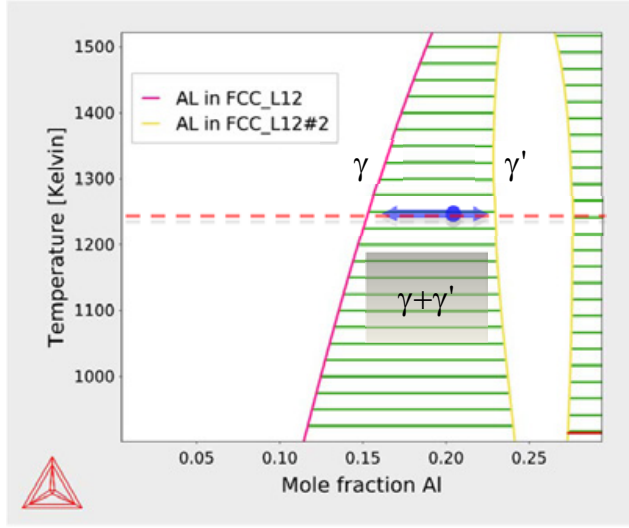

Figure 1. Ni-Al phase diagram showing $\gamma$ and $\gamma^{\prime}$ stable phase regions [21].

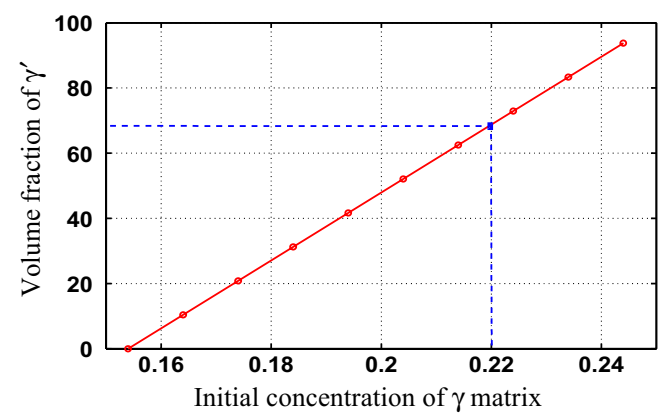

Figure 2. $\gamma^{\prime}$-phase volume fraction vs. initial $\mathrm{Al}$ composition in the $\gamma$-phase in $\mathrm{Ni}-\mathrm{Al}$ superalloy.

difference in coarsening kinetics between two and three dimensions. Thus in order to analyse the coarsening kinetics of $\gamma-\gamma^{\prime}$ microstructure it is important to consider the simulations in $3 \mathrm{D}$ with relatively high number of $\gamma^{\prime}$ precipitates for good statistics. However there are very few published $3 \mathrm{D}$ phase-field simulation results of $\gamma^{\prime}$ precipitation $[16,17]$ and other work concern the morphological evolution of $\gamma^{\prime}$ particles [3]. One could also find very few attempts involving stochastic methods such as Monte Carlo approach but on relatively small-scale simulation $[18,19]$. Cellular Automaton are frequently applied in the field of solidification and grain growth for superalloys [20]. The authors did not find any application to precipitation including misfit strain in large scale mesoscopic simulations. In this study in order to achieve reliable statistics for the coarsening kinetics of $\gamma^{\prime}$ particles we performed the simulation in the domain of $256 \times$ $256 \times 256$ discreet grid points with the grid spacing of $10 \mathrm{~nm}$. While the grid spacing is set by the necessary special resolution of microstructure where the size of cuboids is typically of the order of tens to hundreds of $\mathrm{nm}$, the domain size is mainly limited by the computation time.

For numerical simplification we consider a binary Ni-Al system with only two stable phases: $\gamma$ and $\gamma^{\prime}$. The Ni-Al binary phase diagram is shown in Fig. 1 and is obtained using the Thermocalc [22] with the database developed by Dupin [21]. We start our simulation from the state similar to that after solution heat treatment where dissolution of $\gamma^{\prime}$ particles took place and the Al concentration variation in the system is absent. We start
Table 1. Elastic constants used in this work for $\gamma$ and $\gamma^{\prime}, \mathrm{GPa}$ [5].

\begin{tabular}{|l|c|c|c|c|c|}
\hline $\mathrm{C}_{11}^{\gamma^{\prime}}$ & $\mathrm{C}_{12}^{\gamma^{\prime}}$ & $\mathrm{C}_{44}^{\gamma^{\prime}}$ & $\mathrm{C}_{11}^{\gamma}$ & $\mathrm{C}_{12}^{\gamma}$ & $\mathrm{C}_{44}^{\gamma}$ \\
\hline 222.7 & 164.2 & 85.6 & 215.2 & 162 & 77.6 \\
\hline
\end{tabular}

the simulation of precipitation hardening circle by setting the uniform initial composition of $\gamma$-phase to 22 at. \% Al in order to reach the realistic volume fraction of $\gamma^{\prime}$ phase around $70 \%$ (see Fig. 2). The temperature is set constant at $1273 \mathrm{~K}$ according to the experimental precipitation hardening treatment protocol obtained from other internal project also could be referred in [23].

In our simulations $\gamma^{\prime}$-phase is assumed to be stoichiometric with the fixed concentration of 25 at.\% Al. At the initial state the simulation box is fully occupied by the homogenized $\gamma$-phase. The simulation box is posed with periodic conditions. A set of 1000 random $\gamma^{\prime}$-particles with average radius of $80 \mathrm{~nm}$ is introduced into the simulation box. Since in this study we mainly focus on the coarsening kinetics at the later stage of precipitation hardening heat treatment cycle the initial $\gamma^{\prime}$ particles nucleation was not modelled.

It is known that the main factors controlling the morphology of $\gamma-\gamma^{\prime}$ microstructure are the lattice misfit strain between $\gamma$ and $\gamma^{\prime}$ phases and the anisotropy of their elasticity moduli (see Table 1) which lead to misfit stresses during the microstructure evolution resulting in cuboidal shape of precipitates. Since both $\gamma$ and $\gamma^{\prime}$ have FCC structure only volumetric contribution is present in the transformation strain tensor. The value of lattice misfit strain between $\gamma$ and $\gamma^{\prime}$ phases is set to $-0.3 \%$ according to experimental observation [24].

In our simulation we used an interfacial energy of $80 \mathrm{~mJ} / \mathrm{m}^{2}$ [5] between $\gamma$ and $\gamma^{\prime}$ phases which is similar to CMSX-4. In order to maintain the stability of the $\gamma$-channels and prevent the $\gamma^{\prime}$-particles from coalescence as it is the case in the experiment we used the wetting condition at the $\gamma^{\prime}-\gamma-\gamma^{\prime}$ triple junctions which creates repulsion between $\gamma^{\prime}$-particles by setting the interfacial energy between $\gamma^{\prime}$-particles 3 times higher than the value for $\gamma-\gamma^{\prime}$ interface. The interface mobility in all our simulation is taken in the limit of diffusion controlled transformation. The diffusion coefficient of $\mathrm{Al}$ in $\gamma$ matrix employed here is $5.98 \times 10^{-15} \mathrm{~m}^{2} / \mathrm{s}$ [25] which is very similar to CMSX4 alloy. The elastic constants used in our simulations are summarized in Table 1. Most of the material parameters values are taken for an ERBO-1 alloy which is being studied within the SFB-103 Transregio project [26] and is similar to CMSX-4 alloy [5,21,22, 24,25].

\section{Results and discussions}

\subsection{Two-particle interaction}

In order to study the effect of elasticity on cuboidal shape of $\gamma^{\prime}$-particles and also the effect of wetting condition on $\gamma^{\prime}$-particles coarsening a two-particle simulation had been performed. The starting simulation conditions are given in previous section with the only difference in the number of particles and reduced system size. At the beginning 

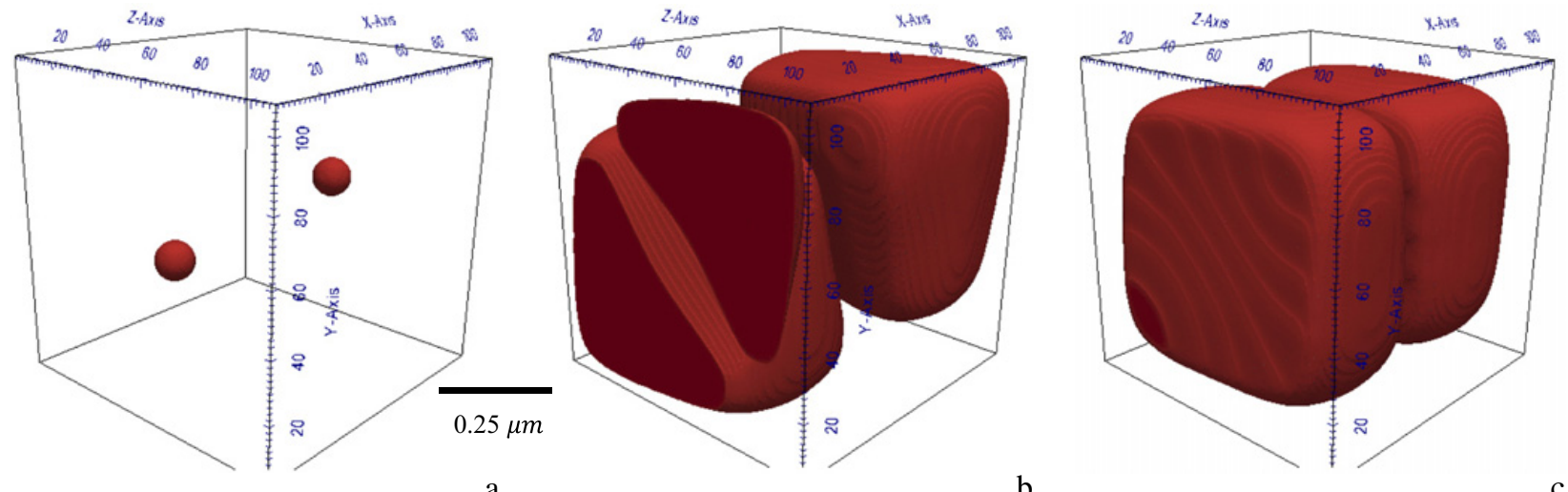

b

Figure 3. Simulation of interaction between two precipitates placed along [111] plane. $\gamma$ phase is made transparent. (a) initial configuration of two precipitate and (c) final coherent state of $\gamma^{\prime}$ precipitates (b) intermediate state where precipitate form an inclined $\gamma$ channel.
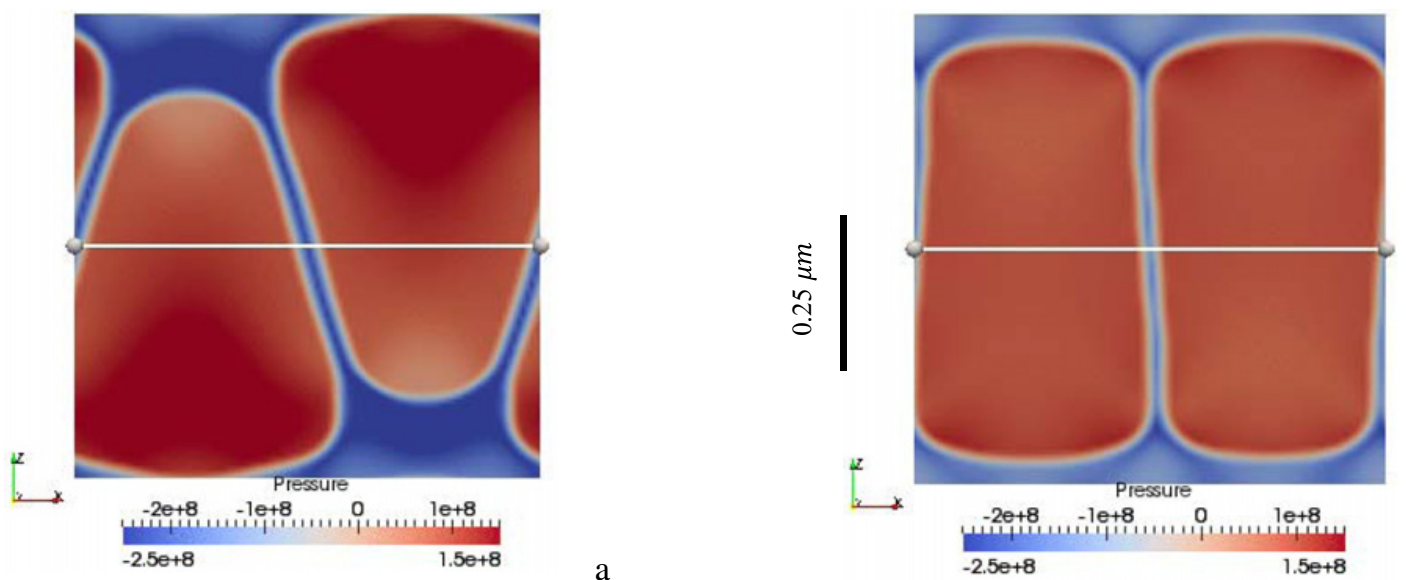

Figure 4. Slice view of stress distribution inside precipitate and matrix. (a) and (b) corresponds to the Figs. $3 \mathrm{~b}$ and c respectively. The sliced view of pressure on simulation domain describes the pressure gradient during the evolution the values are in Pascals.

of the simulation two $\gamma^{\prime}$-precipitates were placed in the simulation box along the main cube diagonal as it is shown in Fig. 3a. As the growth of the particles proceeds, they first increase in size until the chemical driving force becomes negligible (see Fig. 3b) and then start to rearrange due to the elastic energy minimization aligning along the $\mathrm{X}$ crystallographic axis (which coincide with the simulation box's X direction) and simultaneously becoming cuboidal. This simulation clearly emphasizes the effect of elasticity on the cuboidal shape formation of the $\gamma^{\prime}$-particles. The stress field distribution in the simulation domain given in Fig. 4, shows that the stresses are significantly minimized when particles poses cuboidal shape and align themselves along one of the crystallographic axis.

\subsection{Multi-particle microstructure evolution}

In this study we ignore the initial growth regime of the $\gamma^{\prime}$-particles which is shown by the evolution from Fig. 5a (initial state) to the Fig. 5b which indicating intermediate state right after the growth mode. The microstructure shown in Fig. 5b is then used for the study of a coarsening regime of the microstructure evolution. During the initial growth regime the number of $\gamma^{\prime}$-particles remains constant and the cuboidal microstructure consisting of $\gamma^{\prime}$-particles separated by the $\gamma$-channels is formed. Since most of the thermodynamic driving force is already gone in the system due to the growth of the $\gamma^{\prime}$-particles when concentration of $\mathrm{Al}$ in $\gamma$ and $\gamma^{\prime}$ phases approaches the equilibrium one, the curvature driven coarsening regime starts where particles with least curvature have an advantage with respect to the particles with high interface curvature. This leads to the coarsening effect similar to Ostwald ripening but with different coarsening exponent as will be shown in the next section. As already mentioned in previous section in this study we prevent the coalescence of $\gamma^{\prime}$-particles by using the wetting condition at the $\gamma^{\prime}-\gamma-\gamma^{\prime}$ triple junctions, thus only the coarsening phenomenon takes place in our simulations.

In our simulations the volume fraction of the $\gamma^{\prime}$-precipitates is about $64 \%$ which similar to the experimental observation. It is known that with such a high volume fraction of precipitates (above 50\%) the topological inversion should normally take place when precipitates become the matrix phase and the former matrix phase becomes precipitate like. In our simulations as well as in the experiment such topological inversion does not occur due to above mentioned wetting condition set at the 

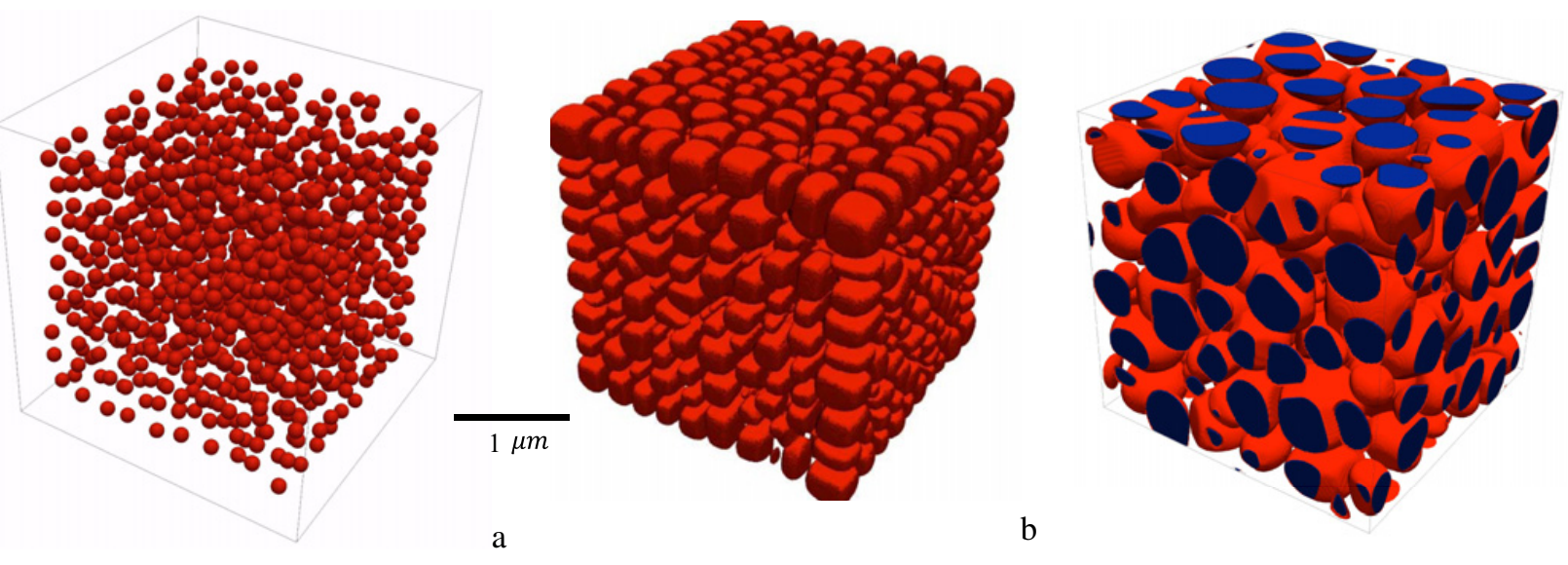

Figure 5. Simulation box of $2.56 \mu \mathrm{m}$ size with periodic boundary condition. For better visualization phases other than $\gamma^{\prime}$ has been made transparent. (a) Initial configuration of 1000 spherical $\gamma^{\prime}$ precipitates (b) microstructure with elastic contribution at $t=70 \mathrm{~s}$ (c) microstructure at $\mathrm{t}=145 \mathrm{~s}$ without the elastic contribution. The faces where the precipitate crosses periodic boundaries is represented in blue.

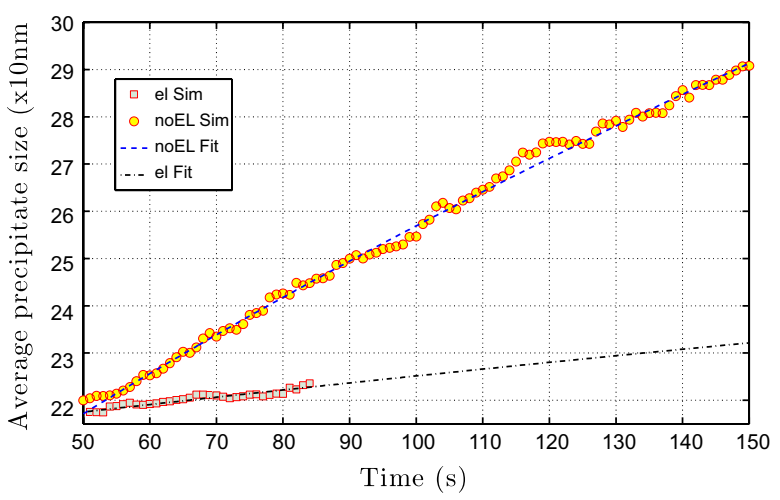

Figure 6. Comparison of average particle size evolution over time in the cases with and without elastic contribution. Dashed and dash-dotted lines show the fits using the power law $\bar{A}^{m}-\bar{A}_{0}^{m}=$ $k t$.

$\gamma^{\prime}-\gamma-\gamma^{\prime}$ triple junctions. Although the exact mechanism leading to the presence of wetting condition at the $\gamma^{\prime}-\gamma-\gamma^{\prime}$ triple junctions is still not well understood, it is nonetheless used as an approximation in our simulations.

\subsection{Coarsening exponent}

The important characteristic of coarsening kinetics is the growth power law for the increase in average length scale. The mean precipitate size $A^{\prime \prime}$ is calculated in the following way.

$$
\bar{A}=\frac{1}{N} \sum_{n} \sqrt[3]{V_{n}}
$$

where $\bar{A}$ is the average cube length computed using average volumes of all precipitates present in the simulation domain, $V_{n}$ is the volume of precipitate $n$ and $N$ is the number of precipitates. In Fig. 6 the circles denote the simulation results without elastic contribution and squares denotes with elastic contribution the dashed and dashed dot lines are the respective fits for simulation results. The initial particle growth regime (first $50 \mathrm{~s}$ of simulation) is excluded from Fig. 6 and also from the fit.

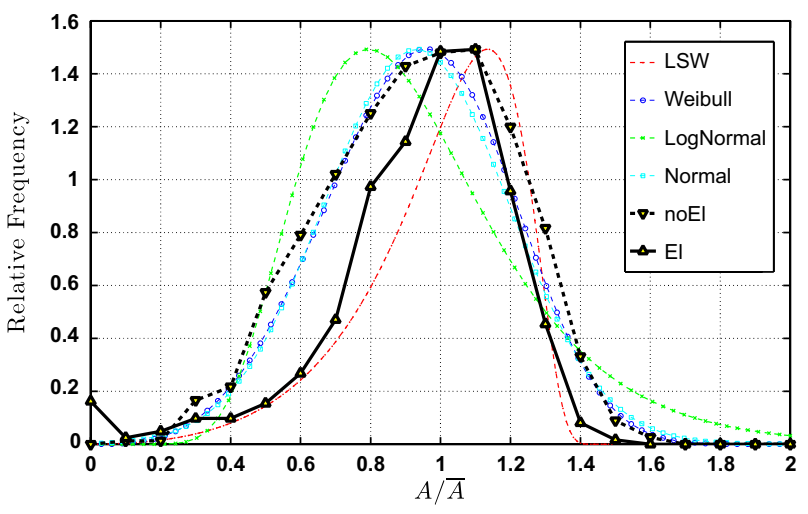

Figure 7. Comparison of PSD with various other distributions at $\mathrm{t}=50 \mathrm{~s}$.

According to our data analysis the coarsening exponent $\mathrm{m}$ in the case without the elastic interaction is near 2.0, while it is equal to 3.6 in the case when the elastic interaction is taken into account. Notably, in most of experiments and other simulations on superalloys the obtained coarsening exponent is around $3[1,8,9,16]$. It is evident that our simulation times are significantly lower than the typical experiment time. Thus one of the explanations of the discrepancy between our results and the experimental observation is the short period of coarsening simulation taken for the exponent evaluation. Another explanation could be the fact that most of the experiments and simulations provide the analysis for $2 \mathrm{D}$ cases where the coarsening exponent is expected to be lower than in 3D.

\subsection{Particle size distribution}

The normalized particle size distribution (PSD) for simulations with and without the elastic interaction is presented in Fig. 7 with a comparison to various known distributions $[2,9,16]$. Figure 7 corresponds to simulation with and without the elastic contribution at $\mathrm{t}=50 \mathrm{~s}$.

The results demonstrate that starting from initially narrow distribution the $\gamma^{\prime}$ PSD evolves towards the Lifshitz, Slyozov and Wagner's (LSW) model [6,7] 
distribution in the case without the elastic interaction. In the case with elastic interaction the PSD lies between the LSW and the normal distribution and is significantly broader than the initial one.

\section{Summary}

In this work we studied the coarsening kinetics of $\gamma^{\prime}$ particles in Ni-Al binary superalloy in three dimensions using the multi phase-field method. Two different cases were numerically simulated in order to investigate the effect of elastic interaction on the coarsening kinetics of $\gamma^{\prime}$-particles. As the result the coarsening exponent for the case with and without the elastic interaction were determined. We show that the elastic interaction due to lattice misfit between $\gamma$ and $\gamma^{\prime}$ phases slows down the coarsening process of $\gamma^{\prime}$-particles. We obtain the coarsening exponent $\mathrm{m}$ close to 2 in the case without the elastic interaction and around 3.6 when taking into account the elastic interaction.

The authors are grateful for funding by the Deutsche Forschungsgemeinschaft (DFG) through Project C5 of the collaborative research center SFB/Transregio 103 on singlecrystal superalloys. We also acknowledge other projects of SFB/Transregio 103 for supporting our simulations with the experimental data. The authors thank Dr.-Ing. Inmaculada LopezGalilea for valuable discussions.

\section{References}

[1] A. Baldan, J. Mater. Sci., vol. 7, pp. 2379-2405, (2002)

[2] Y. Ma and A. Ardell, Scr. Mater, vol. 52, no. 12, pp. 1335-1340, (Jun. 2005)

[3] G. Rubin and a. G. Khachaturyan, Acta Mater, vol. 47, no. 7, pp. 1995-2002, (May 1999)

[4] V. Vaithyanathan and L. Q. Chen, Acta Mater, vol. 50, no. 16, pp. 4061-4073, (Sep. 2002)

[5] J. Kundin, L. Mushongera, T. Goehler, and H. Emmerich, Acta Mater, vol. 60, no. 9, pp. 37583772, (May 2012)
[6] I. Lifshitz and V. Slyozov, J. Phys. Chem. Solids, vol. 19, no. 1, pp. 35-50, (1961)

[7] C. Wagner, Z Elektrochem, vol. 65, p. 581, (1961)

[8] A. J. Ardell and V. Ozolins, Nat. Mater., vol. 4, no. 4, pp. 309-16, (Apr. 2005)

[9] A. M. Ges, O. Fornaro, and H. a. Palacio, Mater. Sci. Eng. A, vol. 458, no. 1-2, pp. 96-100, (Jun. 2007)

[10] R. D. Kamachali and I. Steinbach, Acta Mater., vol. 60, no. 6-7, pp. 2719-2728, (Apr. 2012)

[11] M. Hillert, Acta Met., vol. 13, pp. 227-236, (1965)

[12] [Online]. Available: www.OpenPhase.de

[13] I. Steinbach, Model. Simul. Mater. Sci. Eng., vol. 17, no. 7, p. 073001, (Oct. 2009)

[14] I. Steinbach, Аnnu. Rev. Mater. Res., vol. 43, no. 1, pp. 89-107, (Jul. 2013)

[15] J. Boisse, N. Lecoq, R. Patte, and H. Zapolsky, Acta Mater, vol. 55, no. 18, pp. 6151-6158, (Oct. 2007)

[16] J. Z. Zhu, T. Wang, a. J. Ardell, S. H. Zhou, Z. K. Liu, and L. Q. Chen, Acta Mater., vol. 52, no. 9, pp. 2837-2845, (May 2004)

[17] Y. H. Wen, J. P. Simmons, and C. Woodward, Model. Simul. Mater. Sci. Eng., vol. 18, no. 5, p. 055002, (Jul. 2010)

[18] J. Gayda and D. J. Srolovitz, Acta Metall., vol. 37, no. 2, pp. 641-650, (Feb. 1989)

[19] J. L. Vallés and D. J. Arrell, Acta Metall. Mater, vol. 42, no. 9, pp. 2999-3008, (Sep. 1994)

[20] X. Zhan, Y. Wei, and Z. Dong, J. Mater. Process. Technol., vol. 208, no. 1-3, pp. 1-8, (Nov. 2008)

[21] N. Dupin, I. Ansara, and B. Sundman, Calphad, vol. 25, no. 2, pp. 279-298, (2001)

[22] [Online]. Available: www.thermocalc.com

[23] M. Gebura and J. Lapin, 17th Int. Conf. Met., pp. 1-8, (2008)

[24] A. Kamara, A. Ardell, and C. Wagner, Metall. Mater., vol. 27, no. 10, pp. 2888-2896, (Oct. 1996)

[25] L. Zhang, I. Steinbach, and Y. Du, Int. J. Mater. Res. (formerly Zeitschrift fuer Met., vol. 102, no. 04, pp. 371-380, (Apr. 2011)

[26] [Online]. Available: http://www.sfb-transregio103.de 